\title{
MedienPädagogik
}

www. medienpaed.com

Zeitschrift für

Theorie und Praxis

der Medienbildung

ISSN 1424-3636

Themenheft Nr. 18: Neue Medien und individuelle Leistungsdarstellung -

Möglichkeiten und Grenzen von ePortfolios und eAssessments

\section{E-Portfolios in berufsbegleitenden Studiengängen zu Neuen Medien}

Klaus Himpsl

\begin{abstract}
Der Beitrag stellt das Konzept einer E-Portfolio-Implementierung in einem berufsbegleitenden, postgradualen Masterstudium für eine medienaffine Zielgruppe dar und beschreibt dieses auf mehreren Gestaltungsebenen, vom Curriculum über das Lehr-/Lernkonzept und die verwendeten Technologien bis hin zum Assessment. Für das bessere Verständnis wird zunächst der Studiengang «MA eEducation» vorgestellt und das Blended-Learning-Arrangement beschrieben. Ausgangspunkt für die E-Portfolio-Implementierung ist eine neu entwickelte Taxonomie für E-Portfolios, nach deren Typologie eine Kombination aus Reflexions-, Beurteilungs- und Präsentationsportfolio gewählt wird. Auf der Grundlage der pädagogischen Vorüberlegungen wird der Implementierungsprozess, das Portfoliokonzept und dessen softwaretechnische Umsetzung erläutert, wobei auch auf Fragen des Assessments und des Peer-Feedbacks eingegangen wird.
\end{abstract}

Das Department für Interaktive Medien und Bildungstechnologien (IMB) der Donau-Universität Krems hat sein postgraduales, berufsbegleitendes Masterstudienangebot im Jahr 2007 einer grundlegenden Reform unterzogen, die hinsichtlich der didaktischen Gestaltung der Lehrgänge mehrere Ebenen umfasst (vgl. Abb. 1).

Den bildungspolitischen Rahmen bilden dabei die Erklärungen von Bologna ${ }^{1}$ und Lissabon², zu deren Zielerreichung sich die Donau-Universität Krems als Universität für Weiterbildung mit internationaler Ausrichtung verpflichtet hat.

\section{Der Masterstudiengang «MA eEducation»}

Die Lehrgangsreform wird am Beispiel des Studienganges «MA eEducation» erklärt, der den früheren Lehrgang «Educational Technology» im Sommer 2007 ersetzte. Der Studiengang richtet sich an Lehrende in allen Bildungssektoren und verfolgt das Ziel, «Educational Technologists» auszubilden - ein Begriff, der als

\footnotetext{
Der Europäische Hochschulraum - Gemeinsame Erklärung der Europäischen Bildungsminister, 19. Juni 1999, Bologna, vgl. http://www.bmwf.gv.at/fileadmin/user_upload/europa/bologna/bologna_ dt.pdf

2 Europäischer Rat Lissabon 23-24.03.2000: Schlussfolgerungen des Vorsitzes, vgl. http://www.europarl.europa.eu/summits/lis1_de.htm\#l
} 


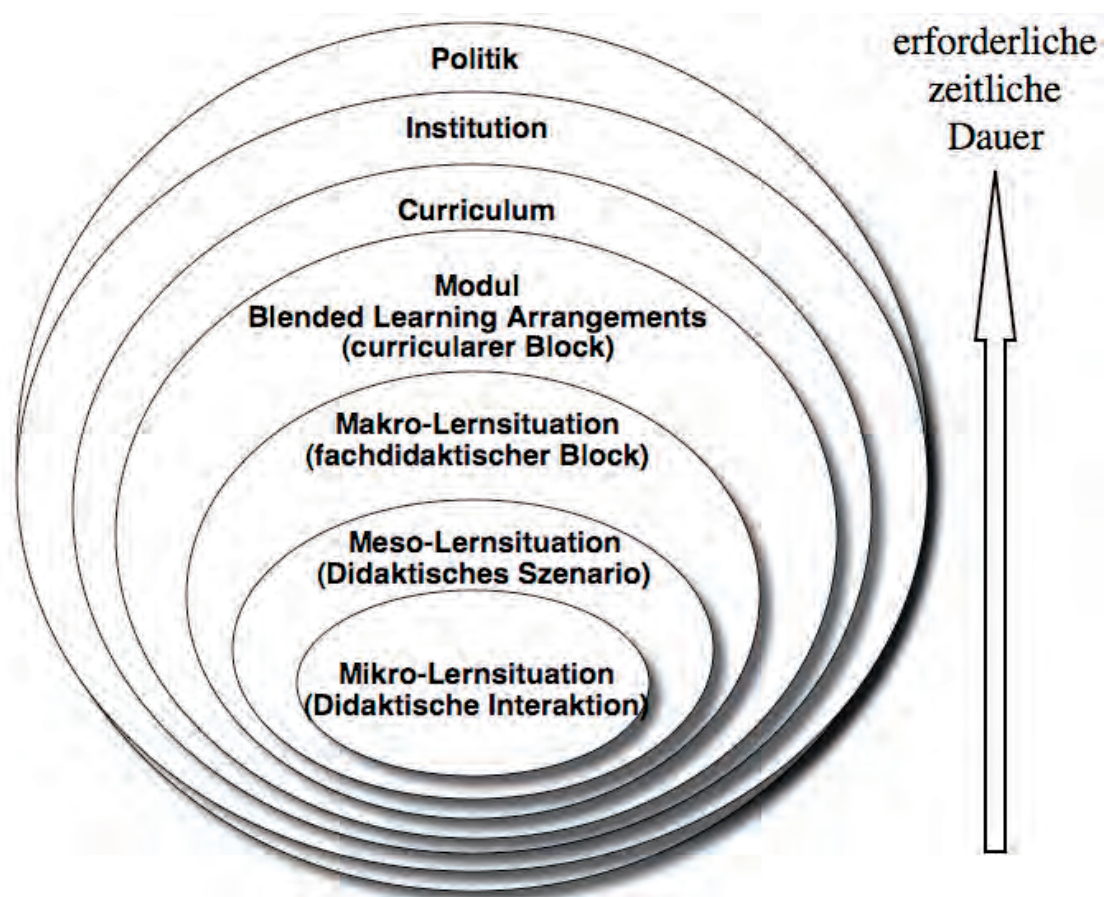

Abbildung 1: Inklusive Hierarchie der didaktischen Gestaltungsebenen (Baumgartner 2008a)

solcher im angloamerikanischen Sprachraum weit verbreitet ist. Dazu werden in $\S 1$ des Curriculums folgende Weiterbildungsziele formuliert ${ }^{3}$ :

1. Die Studierenden werden befähigt, technologieunterstützte Lehr- und Lernangebote wissenschaftlich fundiert zu konzipieren, zu gestalten, zu analysieren und zu evaluieren sowie die erworbenen Kenntnisse in die berufliche Praxis zu transferieren.

2. Die Studierenden werden mit theoretischen Konzepten mediengestützter Bildungsprozesse und bildungstechnologischen Entwicklungen so weit vertraut gemacht, dass sie in die Lage versetzt werden, neue Entwicklungen technologieunterstützter Lehr- und Lernszenarien eigenständig kritisch zu analysieren und zu reflektieren und in die berufspraktische Arbeit zu integrieren.

3. Ziel des Lehrganges ist der Erwerb wissenschaftlicher Forschungs- und Handlungskompetenz auf Basis der Vermittlung pädagogischer, psychologischer, soziologischer und informationstechnologischer Erkenntnisse.

4. Ein wesentliches Charakteristikum des Studienangebotes ist ein interdisziplinärer Zugang, der es ermöglicht, das Thema «eEducation» aus der Perspektive verschiedener wissenschaftlicher Disziplinen zu behandeln und zu diskutieren.

3 Das Curriculum des Lehrganges ist unter http://www. donau-uni.ac.at/eeducation abrufbar. 
Die Annäherung an das zentrale Lehrgangsthema «E-Learning» aus verschiedenen wissenschaftlichen Perspektiven findet sich in der weiteren Ausgestaltung des Curriculums in Form von Modulen wieder.

\section{Modularisierung und Kompetenzorientierung}

Die nationale (österreichische) «Bologna Follow-up Gruppe» hat im Auftrag des österreichischen Bundesministeriums für Wissenschaft und Forschung (bmwf) Empfehlungen für die modulare Gestaltung von Lehrplänen ausgearbeitet (Bacher 2006, S. 1). Nach der Definition dieser Arbeitsgruppe «umfasst ein Modul einen in sich abgeschlossenen, formal strukturierten Lernprozess mit

- thematisch bestimmtem Lernen und Lehren

- festgelegten, kohärenten Lernergebnissen

- vorgegebener Arbeitsbelastung der Studierenden (ausgedrückt in ECTS credits) und

- eindeutigen und transparenten Beurteilungskriterien.»

Die Modulbildung setze nach Bacher auch die Definition der angestrebten Lernergebnisse und der zu erwerbenden Fähigkeiten und Kompetenzen voraus und erfordere verbindliche Absprachen über Inhalte und Ziele, den zeitlichen Ablauf und die Art der vorgesehenen Absolvierung. Kritische Stimmen aus den Reihen der Pädagogik sehen die Gefahr, dass durch die Modularisierung einem stark verkürzten und vereinfachten Kompetenzbegriff Vorschub geleistet würde, nämlich dass die Module wie in einem Baukastensystem in beliebiger Reihenfolge durchlaufen werden könnten und nach deren erfolgreicher Absolvierung die Lernenden gewisse Kompetenzen erworben hätten, die in einem vorgegebenen Kompetenzraster einfach «abgehakt» würden (vgl. hierzu Bakic 2006; Binder 2006). Im Lehrgang «MA eEducation» werden die Module zwar als abgeschlossene Einheiten betrachtet, deren fächerübergreifende Vernetzung allerdings erst das Erreichen der im Curriculum formulierten Weiterbildungsziele sicherstellen soll. Deshalb können die Module auch nicht einzeln und in beliebiger Reihenfolge gewählt und absolviert werden, sondern lediglich in zwei Varianten, innerhalb derer die einzelnen Module aufeinander abgestimmt sind: Die ersten beiden Semester bilden das so genannte «Certified Program» (30 ECTS) mit insgesamt 8 Modulen, die sich an den ersten beiden Weiterbildungszielen aus $\S 1$ des Curriculums orientieren. Im dritten und vierten Semester kommen 9 weitere Module hinzu, die zur Vertiefung hinsichtlich der ersten beiden Ziele und zur Erreichung des dritten Ziels aus $\S 1$ konzipiert sind und zusammen mit dem «Certified Program» das komplette Masterprogramm (90 ECTS) bilden (siehe Abb. 2). 
Für den Umfang der meisten Module wurde eine Grösse von 3 ECTS gewählt, was in etwa einem «studentischen Workload» von 75 Stunden entspricht ${ }^{4}$. Diese Grösse hat sich bisher als günstiger Kompromiss erwiesen, denn die Module sind einerseits gross genug, um eine Vertiefung in die jeweiligen Problemfelder zuzulassen, andererseits so klein, dass sie leicht innerhalb eines Semesters absolviert werden können.

Für die Konzeption des Modulablaufes wurden neben den Bologna-Empfehlungen auch die zusätzlichen Anforderungen berücksichtigt, die ein berufsbegleitendes Studium gegenüber einem Vollzeitstudium an die Studierenden stellt. So soll das Lernpensum neben dem eigenen Beruf bewältigbar sein, ohne dass sich gleichzeitig das Masterstudium über viele Jahre erstreckt. Ausserdem wünschen sich Berufstätige eine hohe zeitliche Flexibilität hinsichtlich der für das Studium zu erledigenden Aufgaben, die bei Präsenzveranstaltungen vor Ort nicht gewähr-

\section{eEducation Certified Program}

\section{eEducation Master of Arts}

\section{1. und 2. Semester}

\section{Bildungstechno-} logie (6 ECTS)

Didaktisches

Design (6 ECTS)

Multimedia

Design

Digitale Mediensozialisation
Medientechnologi sche Grundlagen und Standards

Kognitions- und Motivationspsychologie

\section{Medienpädagogik} und-didaktik

Kommunikationstheorie

\section{3. und 4. Semester}

Bildungstechnologie Vertiefung

Didaktisches Design Vertiefung

Wissensmanagement und eLearning

Corporate eLearning
Einführung in das wissenschaftliche Arbeiten

\section{Forschungs-} methoden

Master Thesis (24 ECTS)

Wissenschaftstheorie

Projekt (15 ECTS)

Abbildung 2: Modularer Aufbau des Studienganges eEducation - Module ohne Zahlenangabe umfassen jeweils 3 ECTS.

leistet werden kann - diese müssen zu vorher festgelegten Terminen stattfinden. Diese Faktoren berücksichtigend wurde die Präsenzzeit vor Ort gegenüber dem früheren Lehrgangskonzept deutlich verringert und stattdessen ein BlendendLearning-Arrangement eingeführt.

4 In Österreich ist ein Umrechnungsfaktor von $1 \mathrm{ECTS}=25$ Stunden üblich, anders als z. B. in Deutschland, wo mit 30 Stunden gerechnet wird. 


\section{Blended-Learning-Arrangement}

Blended Learning bedeutet wörtlich «vermischtes Lernen», wobei traditionellerweise die Mischung von Phasen der Präsenz mit Phasen des E-Learnings gemeint ist (Reinmann-Rothmeier 2003, S. 19). Die freiere deutsche Entsprechung «integriertes Lernen» verweist auf ein integriertes Lernkonzept, das die heute verfügbaren Möglichkeiten der Vernetzung über Internet oder Intranet in Verbindung mit «klassischen» Lernmethoden und -medien einem sinnvollen Lernarrangement optimal nutzt (Werner Sauter, Annette Sauter, \& Bender 2004, S. 68). Aber was ist ein «sinnvolles Arrangement» und wie wird es optimal genutzt?

Baumgartner betont, dass bei der didaktischen Konzeption eines ganzheitlichen Lernprozesses auch die individuelle Auseinandersetzung der Lernenden mit dem Lernmaterial zu berücksichtigen sei (Baumgartner 2008a, S. 11). Bei der Modulplanung werden diese so genannten Selbstlernphasen mit Präsenzunterricht und betreutem Online-Lernen kombiniert, wobei im didaktischen Design neben Reihenfolge und Dauer insbesondere die Schnittstellen zwischen den einzelnen Bestandteilen zu berücksichtigen seien.

Im Lehrgang eEducation, bei dem Inhalt und Methode stark verschmelzen und die Zielgruppe eine hohe Medienaffinität mitbringt, kann der Anteil an Präsenzeinheiten verhältnismässig gering gewählt werden. Bei einem 3-ECTS-Modul wurde dieser auf 10 Stunden festgelegt, was einem Anteil von knapp über 13\% entspricht. Für eine übliche Modulgesamtdauer von etwa vier Monaten gibt es verschiedene Modelle zur Anordnung und unterschiedlichen Gewichtung der drei Anteile Präsenzlernen, betreutes Online-Lernen und Selbstlernen (Sankofi \& Szucsich 2007, S. 63). Für den Lehrgang eEducation wird ein Ablaufmodell gewählt, das die Anordnung eines Präsenztages in der Mitte des viermonatigen Modulzeitraums vorsieht und die restliche Zeit etwa gleichmässig auf betreutes Online-Lernen und Selbstlernphasen aufteilt.

Für die konkrete Ablaufplanung werden Lernziele auf Basis der Bloomschen Taxonomie in der überarbeiteten Fassung von Anderson \& Krathwohl festgelegt (Anderson \& Krathwohl 2001). Lernziele bestehen danach immer aus einem Substantiv, das die Wissensdimension beschreibt (Fakten, Begriffe, Prozeduren, Metakognition), und einem Verb für die kognitive Prozessdimension (erinnern, verstehen, anwenden, analysieren, bewerten, erzeugen), wobei die Stufen als inclusive Hierarchie verstanden werden müssen.

Jedes Lernziel wird auf dem Raster aus 24 Feldern eingeordnet, gleichzeitig werden zugehörige Lernaktivitäten geplant, Lernergebnisse festgelegt und Evaluierungsmethoden zur Überprüfung der Lernzielerreichung generiert (Forehand 2005). Aus der Lernzielfestlegung ergeben sich somit auch Hinweise auf die didaktische Gestaltung des Blended Learning Arrangements und auf die Mischungsverhältnisse der verschiedenen Modi, denn bei höheren kognitiven Prozessen in der Anderson \& Krathwohl Taxonomie (AKT) steigt die Bedeutung des betreuten Lernens und 
der begleitenden Lehrperson, egal ob in Präsenz oder in den E-Learning-Phasen (Baumgartner 2008a, S. 14). Abbildung 3 zeigt prototypische Abläufe des BlendedLearning-Moduls je nach Wahl der AKT-Stufen, wobei im Lehrgang eEducation die erste Variante ohne Präsenzeinheit nicht zum Einsatz kommt.

In den Modulen des Lehrganges «MA eEducation» kommen - je nach gewählten Lernzielen - Mischformen des zweiten und dritten Prototyps zum Einsatz. Da im Studienkonzept die Analyse und kritische Reflexion von technologieunterstützten Lernprozessen sowie deren Übertragbarkeit in die eigene berufliche Praxis im Vordergrund stehen, finden sich viele Lernziele - insbesondere für die OnlineNachphase nach dem Präsenzworkshop - im rechten unteren Bereich der AK-Taxonomie wieder (vgl. Abb. 3): Zur Vertiefung sollen die Studierenden prozedurales und metakognitives Wissen in den Bereichen Analysieren, Evaluieren und Kreieren erwerben und unter Beweis stellen, wofür geschlossene Testformen kaum geeignet sind (Forehand 2005).

Zur Auswahl der geeigneten Leistungsnachweise orientiert sich das IMB am Modell der Arbeitsstelle für Hochschuldidaktik AfH der Universität Zürich (siehe Abb. 4). Dieses Modell stellt Lehren, Lernen und Beurteilen in einem Regelkreis dar, wobei der Leistungsnachweis in das Zentrum gerückt wird - er ist wichtige Orientierungshilfe für sowohl die Dozierenden als auch die Studierenden.

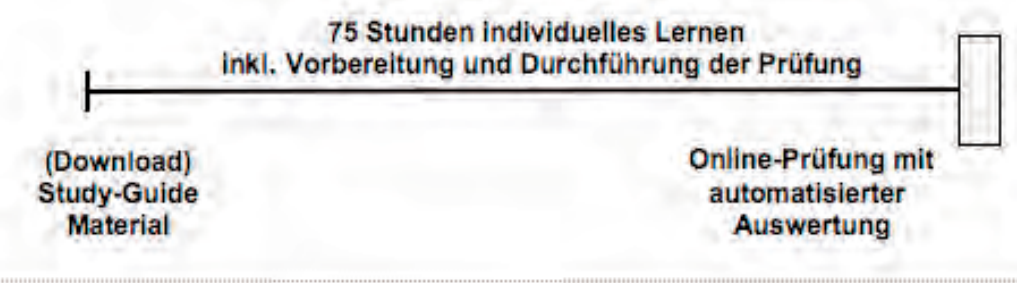

Study-Guide

\begin{tabular}{c}
$\begin{array}{c}\text { Study-Guide } \\
\text { Diskussionsforum, Betreuung } \\
\text { Monat -2 }\end{array}$ Modustart \\
\hline $15 \mathrm{~h}$
\end{tabular}

AKT 1-2: Erinnern, Verstehen

Niedrige Lernzlele sind relativ gut für Selbstlernen und automatislertes ELearning geeignet. Meistens genügt vorbereitetes und automatisch präsentiertes Feedback.

\section{AKT 3-4: Anwenden, Analysieren}

Erfordert im allgemeinen bereits eine inhaltliche tutorielle bzw. moderierende Betreuung, die allerdings noch kein extrem hohes Anspruchsniveau aufweisen muss.

\section{AKT 5-6: Bewerten, Konstruieren}

\section{Arbeitsaufträge} Gruppenbildung, Begleitung

\begin{tabular}{|c|c|c|c|}
\hline \multicolumn{2}{|c|}{ Gruppenbildung, Begleitung } & \multicolumn{2}{|c|}{ Gruppenbildung, Begleitung } \\
\hline Monat -2 & Monat -1 & Monat 1 & Monat 2 \\
\hline $15 \mathrm{~h}$ & $20 \mathrm{~h}$ & $15 \mathrm{~h}$ & $15 \mathrm{~h}$ \\
\hline Modustart & Pr: & Oh) & Modula \\
\hline
\end{tabular}

Es muss neben der Präsenzphase eine intensive und auch inhaltlich anspruchsvolle Betreuung (Coaching, Mentoring) der E-Learning Phasen angeboten werden.

Abbildung 3: Beispiele für Blended Learning Arrangements in Abhängigkeit des gewählten Lernziels nach Baumgartner (2008a). 
Ergänzend zu diesem Regelkreis werden im Dossier «Leistungsnachweise in modularisierten Studiengängen» Leitfragen für Lehrende formuliert: Lehr-Lernkonzeption, Lernstrategien der Studierenden, Lernziele und Kompetenznachweise, Anforderungen (Gütekriterien und der richtige Zeitpunkt) sowie Beurteilung und Rückmeldung (Arbeitsstelle für Hochschuldidaktik der Universität Zürich 2007, S. 19). Eine Analyse der Modulplanungen des Lehrganges «MA eEducation» mit Hilfe dieser Leitfragen ergab verschiedenste Vorschläge von Leistungsnachweisen, die zu den einzelnen Lernzielen passen, wobei die Typen «Lernjournal» und «Portfolio» gehäuft auftraten. Andere passende Prüfungsformen wie "Schriftliche Arbeiten», «Posterpräsentationen», «Gruppenprüfungen», «Wiederkehrende Pflichtnachweise wie Protokolle oder Übungen», «Forumsbeiträge im Netz» lassen sich gut in das Portfolio integrieren. Prüfungsformen, die viel Präsenzzeit vor Ort beanspruchen, wurden aufgrund der reduzierten Präsenzzeiten im Blended-Learning-Konzept auf wenige mündliche Prüfungen an der Universität beschränkt.

In der elektronischen Form erfüllt das Portfolio einen mehrfachen Zweck. Einerseits ist es als Reflexionsportfolio gut geeignet, metakognitive Kompetenzen zu fördern

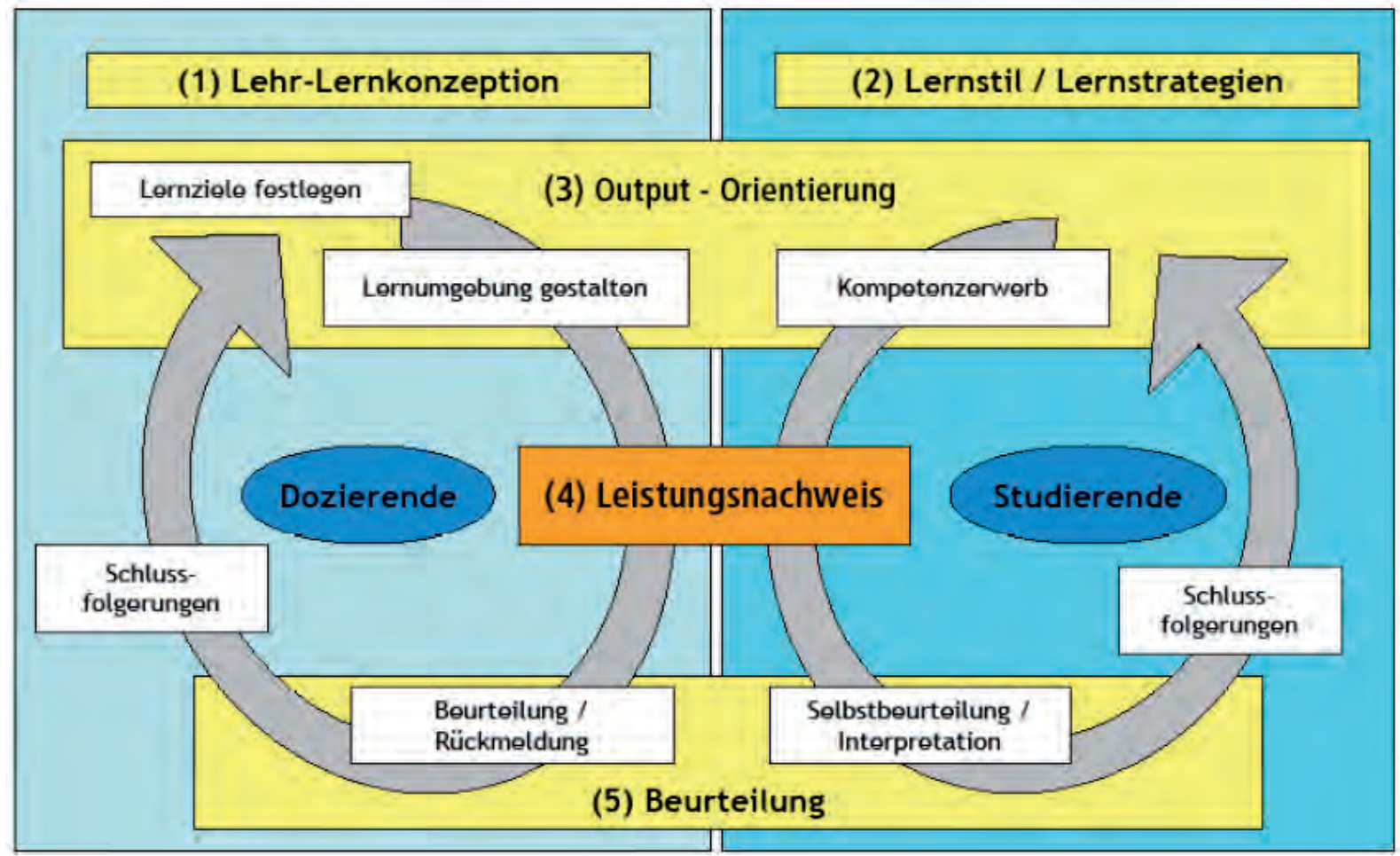

Abbildung 4: Lehren, Lernen und Beurteilen als Regelkreis (Arbeitsstelle für Hochschuldidaktik der Universität Zürich, 2007, S. 8) 
und nachzuweisen (Baumgartner 2005). Andererseits ermöglicht es die Sammlung und übersichtliche Darstellung anderer Leistungsnachweise, die je nach Lernzielen in den jeweiligen Modulen erbracht werden müssen (Hornung-Prähauser u. a. 2007, S. 31). So können z. B. auch Poster aus Gruppenarbeiten in Präsenzworkshops mit Digitalkameras fotografiert oder die Präsentation selbst gefilmt und in das eigene E-Portfolio integriert werden.

\section{Die E-Portfolio-Implementierung am IMB}

Für die Implementierung von E-Portfolios an Hochschulen gibt es in Ländern mit angloamerikanisch geprägter pädagogischer Tradition langjährige Erfahrungen und Modelle, wovon zwei für die Entwicklung des eigenen Konzepts herangezogen wurden.

\section{Implementierungsstrategie}

Das «ePortfolio concept model» der Universität Amsterdam gibt einen einfachen Überblick, welchen Bereichen bei der Implementierung besondere Aufmerksamkeit zukommen sollte (siehe Abb. 5, Veugelers \& Aalderink, 2006).

Nach mehrjährigen Erfahrungen mit E-Portfolios in kompetenzbasierter Ausbildung in zahlreichen Projekten an verschiedenen niederländischen Universitäten werden als «Lessons learned» folgende Empfehlungen zusammengefasst (Veugelers \& Aalderink 2006, S. 4):

1. Die Pädagogik sollte an oberster Stelle stehen.

2. Durchdachte Lernziele und geplante Lernergebnisse sind Ausgangspunkt der Portfolioarbeit.

3. Das zentrale Projektmanagement sollte alle Stakeholder in einer multidisziplinären Herangehensweise einbinden.

4. Die Supportebene ist von grösster Bedeutung, nicht nur hinsichtlich der technischen Infrastruktur.

5. Die eingesetzten Technologien sollten in einer Architektur interoperieren, die lerner/innenzentriert und benutzer/innenfreundlich ist.

Nach diesem Modell (siehe Abb. 5) kommt bei der E-Portfolio-Implementierung am IMB, die zunächst auf Lehrgangsebene gestartet wurde und erst später auf weitere Lehrgänge und Departments ausgedehnt wurde bzw. werden wird, der Lehrgangsleitung die zentrale Rolle zu. Sie stellt die Schnittstelle aller an der Portfolioarbeit beteiligten Personen dar. Für die Bereitstellung der notwendigen Infrastruktur und Gestaltung der Lernumgebung kooperiert sie dabei eng mit dem E-Learning-Center der Universität. Die Integration der Portfolioarbeit in die Lehr-, Lern- und Beurteilungskonzepte der einzelnen Module erfolgt in individuellen Gesprächen mit den Vortragenden, wobei auf Basis des Curriculums und 


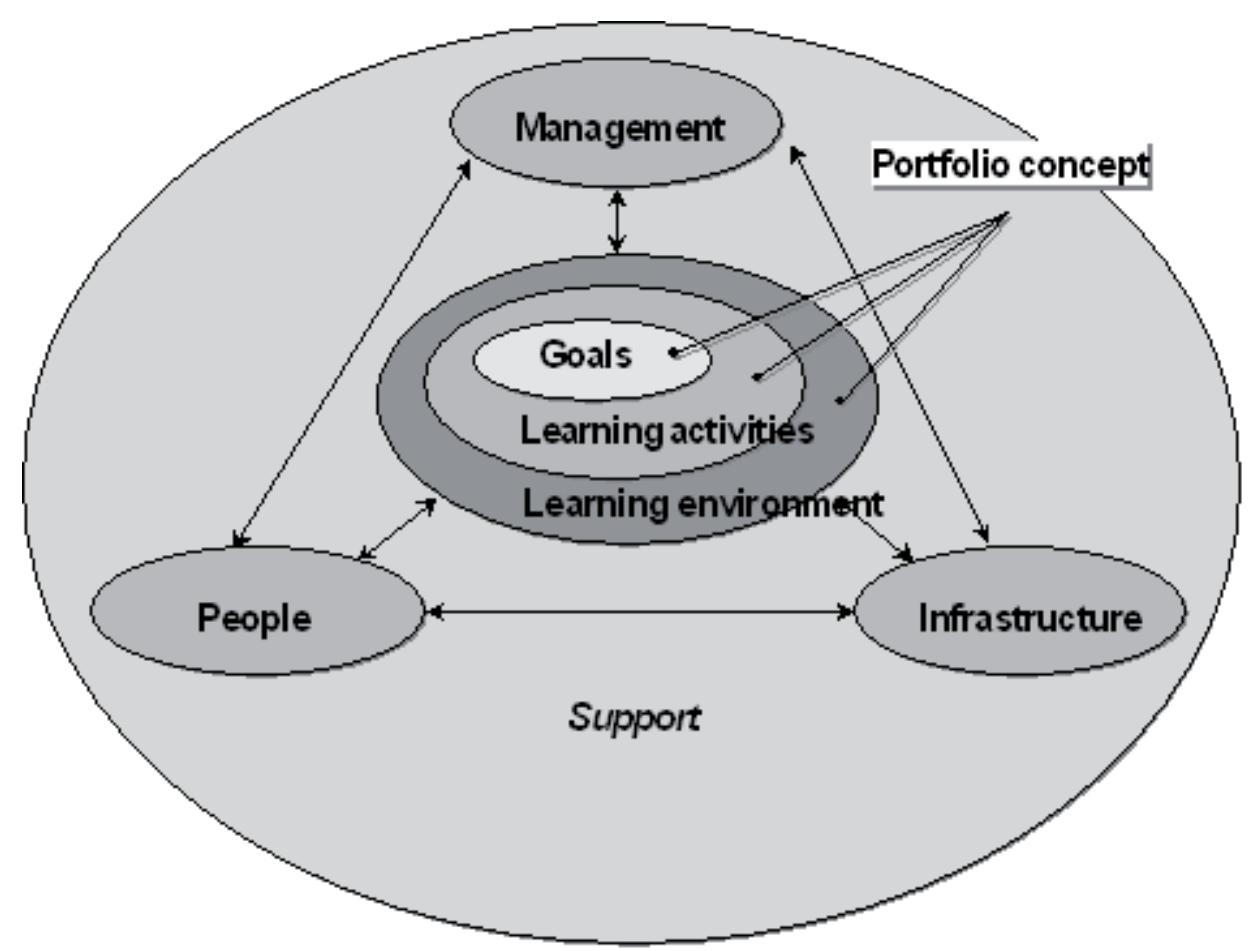

Abbildung 5: «ePortfolio concept model» der Universität Amsterdam (Veugelers \& Aalderink, 2006)

der dort vorliegenden Kompetenzbeschreibungen Lernziele, -aktivitäten und -ergebnisse gemeinsam festgelegt werden. Der Support während der Portfolioarbeit erfolgt auf drei Ebenen:

1. Für Anleitungen und Hilfen wird auf frei zugängliche Materialien der gewählten E-Portfolio-Software Mahara zurückgegriffen, die im Rahmen eines OpenSource-Projektes entwickelt wird. In Zusammenarbeit mit dem E-Learning-Center werden diese Materialien angepasst und ergänzt und - versehen mit einer Creative-Commons-Lizenz - wiederum der Mahara-Community zur Verfügung gestellt.

2. Der technische Support erfolgt durch das E-Learning-Center, in erster Linie über Nachrichtenfunktionen der Software bzw. über E-Mail, aber auch per Telefon.

3. Die inhaltliche, nicht-technische Betreuung der Studierenden erfolgt durch die Lehrgangsleitung sowie durch die Tutoren und Tutorinnen der einzelnen Module.

In den USA gibt es ebenfalls seit vielen Jahren Erfahrungen mit E-Portfolios im Hochschulbereich. «The Pentagonal E-Portfolio Model» fasst die Guidelines für 
Implementierungen von E-Portfolio-Experten und -Expertinnen zusammen und beschreibt Aktivitäten in insgesamt 16 Feldern (siehe Abb. 6, Buzzetto-More \& Alade, 2008, S. 50).

Aus diesem Modell wurde am IMB ein Implementierungsplan über drei Jahrgänge hinweg entwickelt, der folgende Stufen umfasst:

1. Planung und Vorbereitung (Level I, II und III)

2. Erster Jahrgang: Pilotimplementierung in wenigen Modulen des Curriculums, kooperative Entwicklung von Assessmentkriterien, begleitende Evaluation (Level IV und V)

3. Zweiter Jahrgang: Vollimplementierung mit Verankerung des Portfolios im Curriculum, Überarbeitung der Beurteilungskriterien sowie einer umfassenden Evaluation (Level IV und V)

4. Dritter Jahrgang: Re-Design und Weiterentwicklung, Anpassung von Lernzielen, Lernergebnissen, «Performance Indicators» sowie Beurteilungskriterien (Level IV und V)

Aktuell (Dezember 2009) läuft die Vollimplementierung im zweiten Jahrgang, Evaluation und Re-Design sind für 2010 und 2011 geplant. Im Folgenden werden die wesentlichen Punkte auf den ersten drei Stufen des Implementierungsplans dargestellt.

\section{Ausgangspunkt: eine Taxonomie für E-Portfolios}

Neben der Definition der Lernziele und Lernergebnisse sind die genaue Festlegung des E-Portfolio-Zwecks und des E-Portfolio-Typs wichtige Eingangsvoraussetzungen, was sich aufgrund sehr unterschiedlicher Begriffe und Beschreibungssysteme in der einschlägigen Fachliteratur als keine leichte Aufgabe erweist - so identifiziert z. B. Häcker 30 verschiedene Arten von Portfolios im pädagogischen Kontext (Häcker 2007, S. 132). Deshalb wurde im Rahmen des Forschungsprojektes «E-Portfolios an (österreichischen) Hochschulen» eine Taxonomie für E-Portfolios entwickelt (Baumgarnter, Himpsl, \& Zauchner 2009). Zentrales Ergebnis ist eine Einteilung in die drei Grundtypen Reflexionsportfolio, Entwicklungsportfolio und Präsentationsportfolio, die zusätzlich nach zwei weiteren Kriterien differenziert werden:

1. nach Eigentumsrecht: personenbezogen oder organisational

2. nach Orientierung: produktbezogen oder prozessbezogen

So ergeben sich zu jedem der drei Grundtypen jeweils vier Untertypen von Portfolios, also insgesamt 12 Typen von Portfolios (siehe Abb. 7). 


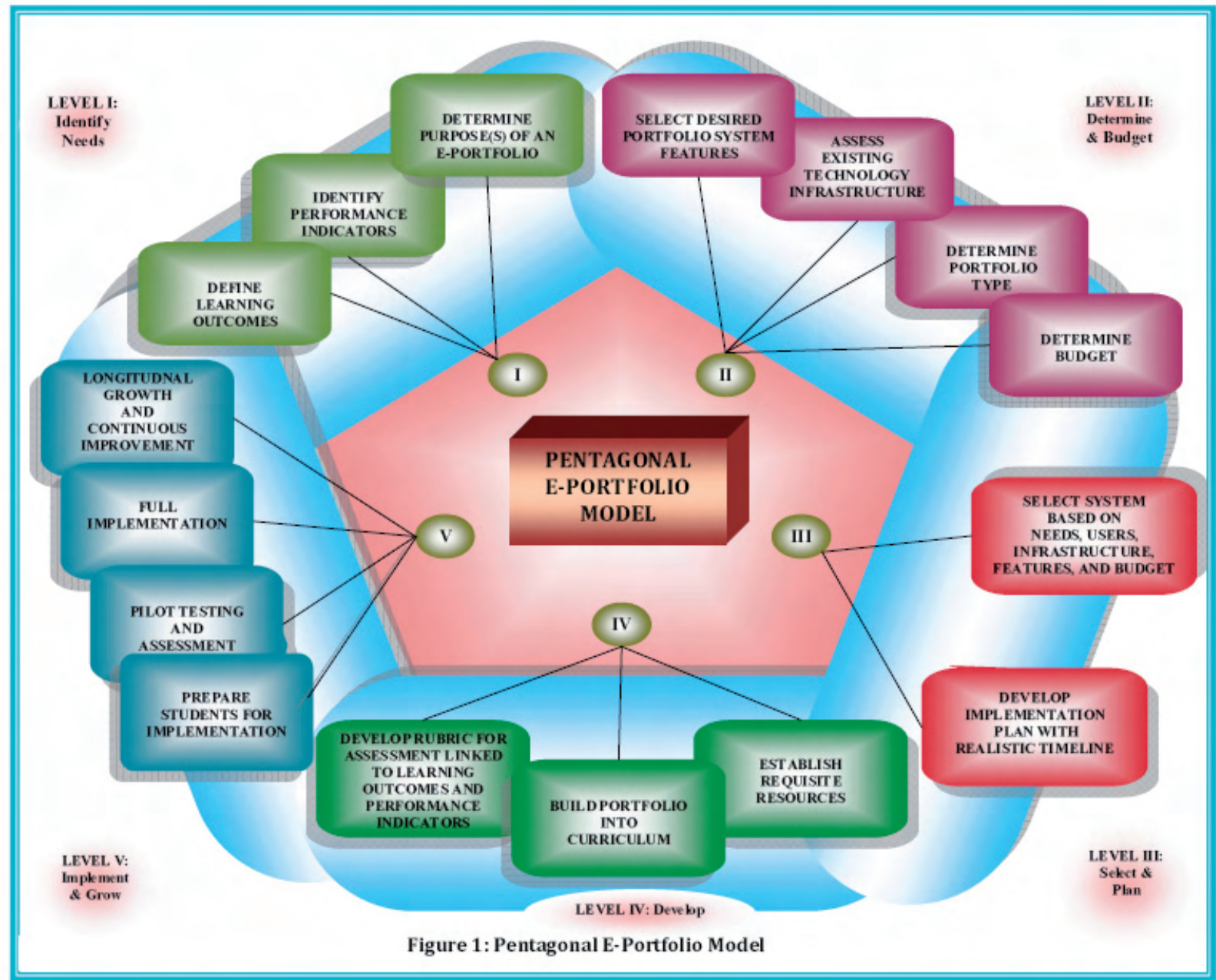

Abbildung 6: «The Pentagonal E-Portfolio Model nach Buzzeto-More \& Alade (2008)

Für die Auswahl des geeigneten Typs am IMB wurde zunächst eine zentrale These aus der Taxonomie für E-Portfolios aufgegriffen (Baumgarnter, Himpsl, \& Zauchner 2009, S. 5):

Für die Unterstützung im Studium machen in erster Linie Reflexionsportfolios - sowohl in der Lern- als auch in der Beurteilungsvariante - Sinn.

Die beiden weiteren Grundtypen, das Entwicklungsportfolio und das Präsentationsportfolio, seien im Hochschulkontext zwar ebenfalls relevant, würden allerdings erst über das Studienende hinaus im Sinne des «Lebenslangen Lernens» an Bedeutung gewinnen. 
Das Reflexionsportfolio kann nach Baumgartner in vier weitere Grundtypen eingeteilt werden, wobei die ersten beiden personenbezogenen Varianten «Lernportfolio» genannt werden und im engeren Sinn der Reflexion des eigenen Lernens dienen (Baumgartner 2008b, S. 51). Bei den beiden organisationsbezogenen Varianten werden die Portfolios innerhalb der Organisation zu Prüfungszwecken zur Beurteilung vorgelegt und Prüfungsportfolio bzw. Curriculumsportfolio genannt, je nachdem, ob und wie stark das Portfolio auf der curricularen Ebene verankert ist (Baumgartner 2008b, S. 53).

Angelehnt an die Ziele des Studienganges wurde für das E-Portfolio-Konzept im Lehrgang «MA eEducation» eine Kombination aus drei Typen gewählt:

1. Das Lernportfolio (personenbezogen, prozessorientiert) wird parallel zur Laufzeit eines Moduls geführt und dient in erster Linie der Reflexion des eigenen Lernprozesses. Es ist deshalb grundsätzlich freiwillig, wird den Studierenden allerdings - eingebettet in ein Peer-Review-Verfahren - empfohlen.

2. Das Curriculumsportfolio (organisationsbezogen, produkt- und prozessorientiert) wird aus dem Lernportfolio heraus gegen Ende der Modullaufzeit erstellt und den Lehrenden zur Beurteilung vorgelegt. Dieses Portfolio ist verpflichtend, die Bewertung der Modulleistung mit einer Ziffernnote nach dem österreichischen Notensystem erfolgt auf Grundlage dieser Portfolioansicht.

3. Das Präsentationsportfolio (personenbezogen, eher produktorientiert) kann von den Studierenden parallel zum Studium als Zusammenschau der Portfolioansichten aus den einzelnen Modulen ohne grossen Zusatzaufwand erstellt werden. Es soll den Absolventen und Absolventinnen neben dem Diploma Supplement als umfassender Nachweis der im Studium erworbenen Kompetenzen dienen und kann bzw. soll auch Ergebnisse informellen Lernens enthalten. Im Sinne des «Lifelong Learning» kann es auch als digitales Bewerbungsportfolio verwendet werden.

Nach der Festlegung des Portfoliozwecks und der Analyse der bestehenden Infrastruktur am Department erfolgte die Auswahl der benötigten Features des EPortfolio-Systems und einer geeigneten technologischen Umsetzung.

\section{Software-Auswahl}

E-Portfolios sind ein neuer Softwaretypus und es ist derzeit noch relativ unklar, welche Funktionen obligatorisch - also die charakteristischen Eigenschaften ausmachen - und welche Funktionen bloss fakultativ («nice to have») sind. Entwürfe für künftige E-Portfolio-Software-Lösungen skizzieren eine verteilte Architektur, die je nach Blickwinkel den organisationalen (Ravet 2007) oder den individuellen Charakter im Sinne eines Personal Learning Environments (Attwell 2007) betonen - technische Realisierungen gibt es derzeit noch nicht. Um von den Vorteilen der 


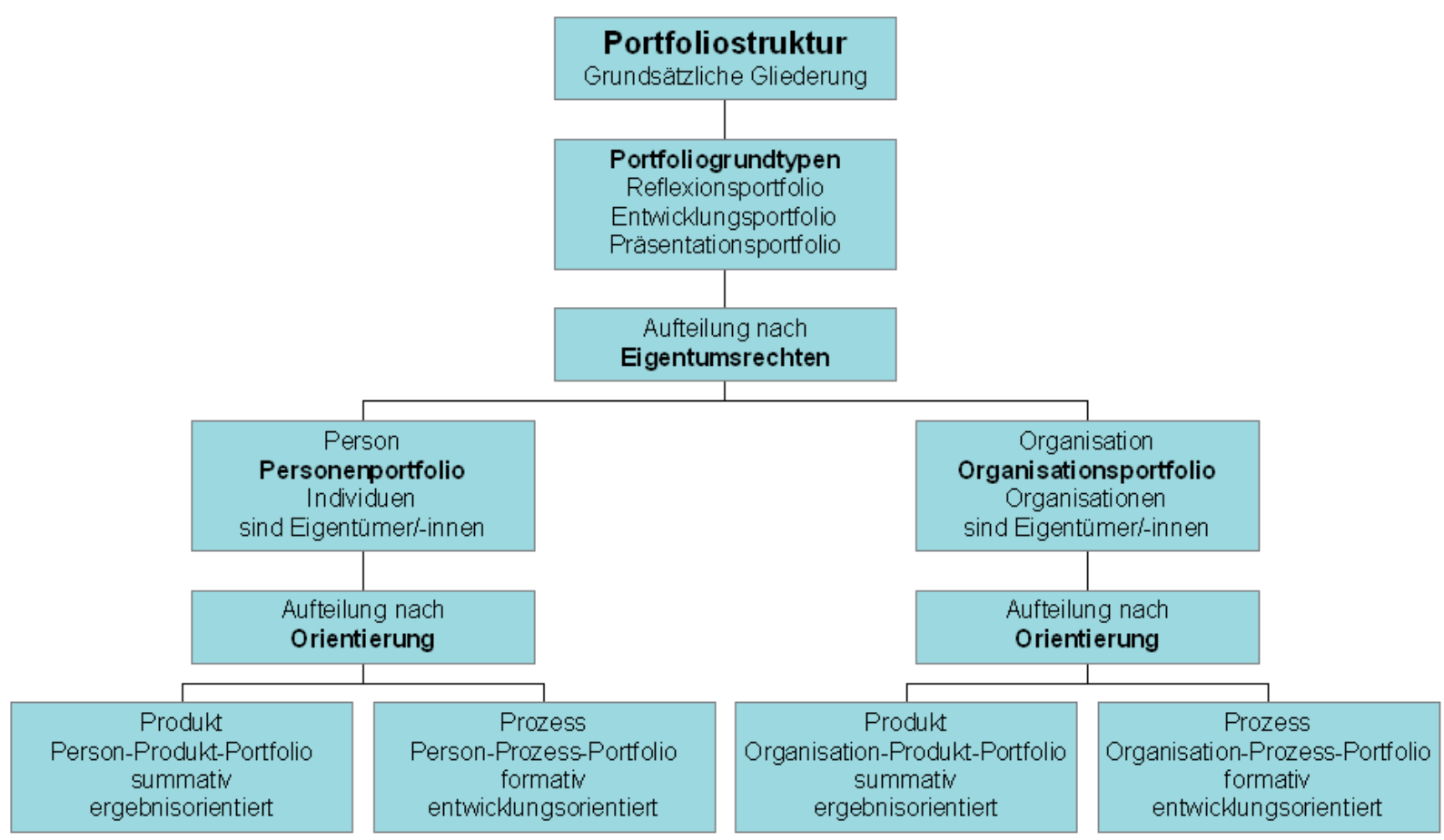

Abbildung 7: Einteilung von E-Portfolio-Typen nach Baumgartner, Himpsl \& Zauchner (2009, S. 4)

Online-Kommunikation und -Kooperation zu profitieren, ist eine webbasierte Lösung zu bevorzugen. Allerdings können Daten, die für eine Verwendung in einem persönlichen Portfolio potentiell in Frage kommen, in ganz verschiedenen Systemen abgelegt werden, wie die Abbildung 8 zeigt:

Welche Art von Software ist für welchen Verwendungszweck eines Portfolios am besten geeignet? Sollen bestehende Lernplattformen (LMS, LCMS), Content-Management-Systeme (CMS) oder Human-Ressource-Management-Systeme (HRMS) für die Portfolioarbeit verwendet werden? Oder ist es geschickter, auf Web-2.0Applikationen zurückzugreifen? Wie ist das Verhältnis von Personal Learning Environment (PLE) und E-Portfolio? Um sich diesen Fragen anzunähern und eine passende Softwarelösung für «MA eEducation» auszuwählen, wurde ein Vorgehen in folgenden Schritten gewählt:

1. Analyse der eigenen technischen Infrastruktur und sonstiger Ressourcen am Department

2. Festlegung von in erster Linie pädagogisch motivierten Prämissen für die Vorauswahl

3. Literaturrecherche und Auswahl eines Systems für den Pilotversuch

4. Durchführung einer umfassenden E-Portfolio-Software-Evaluation im Rahmen des Forschungsprojektes «E-Portfolios an (österreichischen) Hochschulen» 
5. Endgültige Auswahl des geeigneten Systems

Im Lehrgang kommen diverse Webplattformen zum Einsatz, die teilweise selbst gehostet werden, teilweise als frei zur Verfügung stehende kostenlose Services genutzt werden. Auf dem eigenen Server wird das Open-Source-Learning-Management-System Moodle eingesetzt sowie ein internes und ein öffentlich zugängliches Wiki-System, für deren technische Betreuung zwei Administratoren zur Verfügung stehen. Als externe Services werden zum Beispiel Social Bookmarker oder Online-Mindmapping-Software genutzt. Aus der Analyse der eigenen Ressourcen sowie des E-Portfolio-Konzeptes wurden folgende Festlegungen für die Softwareauswahl getroffen:

- Die Entwicklung einer eigenen Speziallösung ist nicht leistbar, die Verwendung einer Eigenentwicklung von anderen Hochschulen, z. B. aus den USA, zu unsicher. Aufgrund der vorhandenen, versierten EDV-Administratoren ist eine Eigeninstallation einer Open-Source-Lösung einem kommerziellen Produkt aus Kostengründen vorzuziehen.

- Die Studierenden müssen selbst den Zugriff auf Teile ihres Portfolios differenziert regeln können: Das Reflexionsportfolio wird völlig intern geführt bzw. lediglich freiwillig einzelnen Peers gezeigt, das Curriculumsportfolio muss in der Lerngruppe zur Verfügung gestellt werden können, das Präsentationsportfolio muss - sofern von den Studierenden gewünscht - auch veröffentlicht werden können.

- Aufgrund des organisationsbezogenen Curriculumsportfolios, das zur Beurteilung vorgelegt werden muss und prüfungsrelevante Leistungen von Studierenden enthält, muss die Institution Zugriff auf die Daten sicherstellen können deshalb scheiden die freie Wahl des Tools durch die Studierenden selbst sowie eine Lösung eines Drittanbieters aus.

- Im Hinblick auf die Verwendung des Portfolios ausserhalb des Studiums im Sinne des «Lifelong Learning» darf die Portfoliolösung nicht zu eng mit den verwendeten Plattformen Moodle und Wiki verbunden sein und muss auch nach Ende des Studiums weiterhin zur Verfügung stehen.

Ausgehend von diesen Grundannahmen wurde im Frühjahr 2007 eine Recherche durchgeführt, wobei insbesondere der von der Salzburg Research Forschungsgesellschaft bereitgestellte Überblick über den E-Portfolio-Software-Markt wertvolle Hinweise lieferte (Hornung-Prähauser et al., 2007, S. 47-104). Die dort vorgestellten Open-Source-Lösungen wurden installiert und getestet, wobei für den Pilotversuch die Wahl auf «Mahara» ${ }^{5}$ fiel. Parallel zu den eigenen Erfahrungen im Pilotversuch

5 Mahara ist ein Open-Source-Produkt, das aus einem Projekt eines Universitätenverbundes in Neuseeland entstanden ist (vgl. http://www.mahara.org). Die vom IMB gehostete Mahara-Plattform ist 


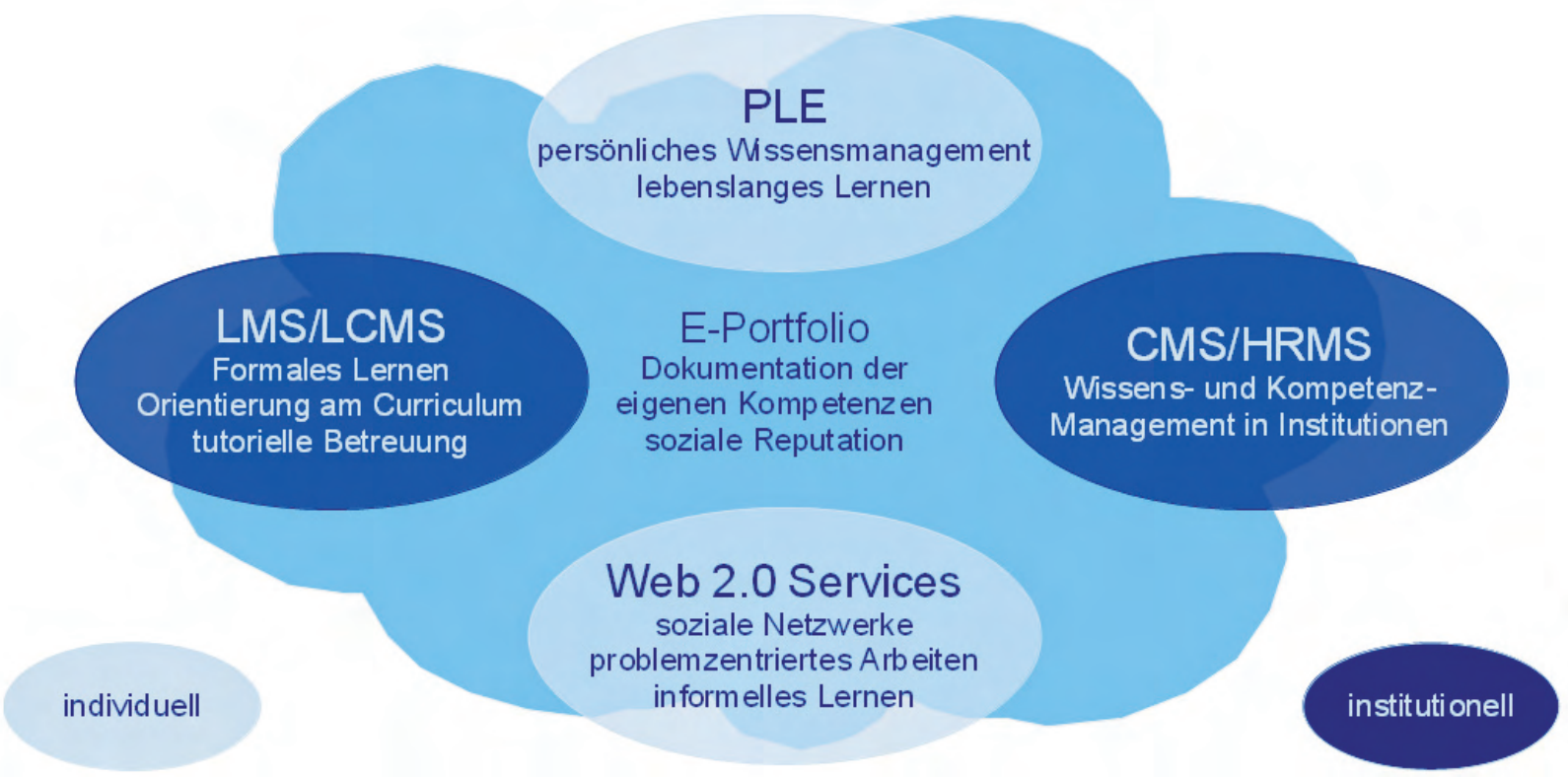

Abbildung 8: Überblick Software-Systeme mit E-Portfolio-Anteilen (eigenes Modell, angelehnt an Erpenbeck \& Sauter, 2007)

wurde im Rahmen des Forschungsprojektes «E-Portfolios an (österreichischen) Hochschulen» eine umfassende E-Portfolio-Software-Evaluation durchgeführt, bei der aus einer Marktübersicht von etwa 60 Anbietern eine "Shortlist» von $12 \mathrm{emp}$ fehlenswerten Produkten entstand (Himpsl \& Baumgartner 2009, S. 25). Innerhallb der Shortlist stellen Mahara und PebblePad die ausgeglichensten Produkte dar, wobei PebblePad ein kommerzielles Produkt ist, bei dem Lizenzgebühren anfallen. Zusammen mit den überwiegend positiven Erfahrungen im Pilotversuch blieb somit Mahara die erste Wahl für die Vollimplementierung.

\section{Das E-Portfolio-Konzept im Studiengang «MA eEducation»}

Aus Sicht des IMB sind die Zielsetzungen beim Lern- und Curriculumsportfolio ein verstärktes Reflektieren der Studierenden über die Lehrinhalte, die vertiefte Auseinandersetzung mit den dargebotenen Aufgabenstellungen, eine stärkere Identifikation der Studierenden mit dem eigenen Lernprozess und daraus resultierend höhere Lernerfolge bezüglich der Thematik und den Zielen des jeweiligen Moduls. Diese lerner/innenzentrierte Auslegung des Portfoliobegriffes ist umso wichtiger, als in der gegenwärtigen E-Portfolio-Literatur und in den zahlreichen Implementierungen in vielen Ländern die curricularen Ziele der Institutionen im Vordergrund zu stehen scheinen. So stellt z. B. Ayala im Frühjahr 2006 nach der Analyse von etwa 300 Fachartikeln zum Thema «E-Portfolio» fest, dass sich mehr als zwei Drittel da-

unter http://www.mahara.at zu erreichen. 
von mit Fragen des Assessments und des Rechenschaftablegens beschäftigen und sogar nur in 5\% der Artikel die Studierenden selbst zu Wort kämen (Ayala 2006, S. 12). Ayala beklagt, dass die Portfolios häufig auf dem Rücken der Studierenden implementiert würden und statt deren Lernerfolge institutionelle Interessen im Vordergrund stünden. Um solchen Tendenzen entgegen zu wirken, wird bei der EPortfolio-Implementierung im Lehrgang «MA eEducation» von Anfang an auf eine hohe Partizipation der Studierenden Wert gelegt.

\section{Einführung von Studierenden und Lehrenden}

Bei der Ausdifferenzierung und Umsetzung des Portfoliokonzeptes orientiert sich das IMB an reformpädagogischen Ansätzen und Werthaltungen, wie sie zum Beispiel im «Handbuch Portfolioarbeit» dargestellt werden (Brunner, Häcker, \& Winter 2006). Auch wenn die Ausführungen dort in erster Linie den schulischen Bereich ansprechen, lassen sich dennoch viele Grundprinzipien auf die Erwachsenenbildung übertragen. Einem berufsbegleitenden, postgradualen Studienangebot angemessen werden die Studierenden als Experten und Expertinnen in bestimmten Feldern angesehen, deren Expertise und Kompetenzen in der Auseinandersetzung mit den Themen des Studiums für die Lerngemeinschaft sehr wertvoll sind, sofern sie eingebracht werden können. Die Portfolioarbeit soll selbstbestimmtes und wechselseitiges Lernen fördern und durch Reflexion und Kommunikation ein Klima des Vertrauens aufbauen. Die Lehrenden sehen sich selbst als Teil der Lerngemeinschaft mit Tutoring- und Coachingfunktionen. Für ein Gelingen der Portfolioarbeit identifiziert Häcker drei Grundsäulen (Häcker 2007):

1. Kommunikation: eine offene Gesprächskultur hinsichtlich der Ziele der Portfolioarbeit, den aktuellen Stand der Arbeiten und der Bewertung, Austausch in der Lerngruppe und mit den Lehrenden, hohe Bedeutung von Feedback

2. Transparenz: Klarheit bei allen Beteiligten über die Ziele der Portfolioarbeit und die Bewertung

3. Partizipation: Einbeziehen der Lernenden bei der Auswahl der Portfolioinhalte, der Festlegung der Auswahlkriterien sowie der Festlegung der Kriterien der Leistungsbeurteilung

Diesen Grundprinzipien folgend wird den Studierenden das Portfoliokonzept zu Beginn des Studiums vorgestellt und in den ersten drei Monaten begleitend zum Modul «Bildungstechnologie» (6 ECTS) eingeführt. Die Einführung gliedert sich in folgende Phasen:

1. Bereitstellung von Materialien über die Lernplattform: 10-seitige Kurzbeschreibung des Konzepts, Online-Tutorials zur Bedienung der Software, weiterführende Literatur 
2. Eintägiger Präsenzworkshop beim Lehrgangsstart: Einführender Vortrag zum Konzept, Übungen dazu, Einführung in die Bedienung der Software, Erarbeitung des Peer-Review-Verfahrens

3. Online-Betreuung bis zum Abschluss des Moduls: Beantwortung von Fragen über ein Diskussionsforum, Begleitung des Peer-Review-Verfahrens, Zwischenfeedback zu einzelnen Lernergebnissen, Zwischen- und Abschlussfeedback zur eingereichten Portfolioansicht

4. Kurzer Abschlussworkshop in Präsenz: Klärung von Fragen, Diskussion der Bewertungskriterien, Festlegung des Peer-Review-Verfahrens für die folgenden Module

Die Erarbeitung des Konzeptes und die Festlegung der «Regeln» für die Portfolioarbeit verlangt in diesem Modul eine intensive Betreuung. Die Vortragenden der weiteren Module profitieren von dieser Vorarbeit, weil den Studierenden der grundlegende Portfolioprozess und die verwendete Software vertraut sind.

Die Lehrenden - viele von ihnen sind externe Lektoren bzw. Lektorinnen - werden in individuellen persönlichen Gesprächen mit der Lehrgangsleitung in das Blended-Learning- und E-Portfoliokonzept eingeführt. Die didaktischen Konzepte und Modulbeschreibungen werden in enger Absprache mit der Lehrgangsleitung entworfen. Zusätzlich erhalten sie Zugriff auf die Online-Umgebung des Moduls «Bildungstechnologie» und können sich so ein Bild über den Portfolioprozess, die Portfolioansichten und das Feedback-Verfahren machen.

\section{Die Umsetzung des Portfoliokonzepts mit Mahara}

Mahara ist eine Open-Source-E-Portfolio-Software mit Social-Networking-Funktionen und verbindet einen individuellen, lerner/innenzentrierten Ansatz mit einem Community-Konzept, d.h., die zur Verfügung gestellten Tools dienen zunächst den Lernenden zur Begleitung des eigenen Lernprozesses und der eigenen Entwicklung, bieten daneben aber auch die Möglichkeit, anderen Usern/Userinnen Zugriff zu erlauben und sich so einem gewissen Personenkreis zu präsentieren. Die wesentlichen Elemente der Software sind:

- ein erweitertes Profil, das ähnlich dem CV des «Europasses» gestaltet ist,

- eine strukturierte Dateiablage mit Tags,

- eine Blog-Funktion, mit der ein oder mehrere Lerntagebücher angelegt werden können, wobei Referenzen auf die Lernprodukte der Dateiablage möglich sind,

- ein Präsentations-Tool, mit dem aus den vorhandenen Artefakten so genannte Views zusammengestellt und einzelnen Usern/Userinnen, Gruppen oder der Öffentlichkeit präsentiert werden können. 
Nach dem Befüllen der Profildaten arbeiten die Studierenden modulweise an einem Lernportfolio, das gegen Ende des Modulzeitraumes in ein Curriculumsportfolio übergeht und zur Prüfung vorgelegt wird (siehe Abbildung 9).

In Anlehnung an die Prozesskomponenten der Portfolioarbeit nach Häcker werden folgende Schritte durchlaufen (Häcker 2007, S. 145):

1. "Context Definition»: Ausgangspunkt für die Portfolioerstellung ist das Curriculum des Studienganges sowie die detailliertere Beschreibung des jeweiligen Moduls, in der zu den übergeordneten Kompetenzen konkrete Lernziele und Lernergebnisse definiert werden. Zweck und Kontext der Portfolioarbeit werden geklärt.

2. "Collection»: Die Studierenden sammeln «Lernprodukte» und legen diese in der Dateiablage von Mahara ab, die ebenso wie eine lokale Festplatte durch Verzeichnisse und Unterverzeichnisse strukturiert werden kann. Lernprodukte können verschiedenste Dateitypen sein: Audio- und Video-Dateien, Textverarbeitungsdokumente, Bilder, Präsentationsfolien etc. Zusätzlich zur Einordnung in das Dateiensystem können die Dokumente mit Stichwörtern («Tags») versehen werden, die sich z. B. auf ein bestimmtes Lernziel beziehen.

3. «Reflection»: Zu jedem abgelegten Lernprodukt wird eine Notiz im Lernjournal eingetragen, das in Mahara Blog genannt wird. Durch diesen Eintrag wird das Lernprodukt in seinen Entstehungskontext eingeordnet, wobei Fragen wie «Wie bin ich an die Aufgabe herangegangen?», «Welche Schwierigkeiten sind aufgetreten?», «Wer oder was hat mir bei der Lösung geholfen?» usw. beantwortet werden. Schliesslich wird durch einen Hyperlink eine Verbindung zwischen dem Blogposting und dem Lernprodukt in der Dateiablage hergestellt. Dieses Reflexionsportfolio kann über eine Ansicht («View») den Peers zugänglich gemacht werden, so dass über die Kommentarfunktion der Software zu einzelnen Tagebucheinträgen Feedback abgegeben werden kann.

4. «Selection»: Vor dem Abgabetermin zum Modulende erfolgt die Zusammenstellung des Curriculumsportfolios. Der/die Lernende wählt diejenigen Lernprodukte aus, die am besten das Erreichen der Lernziele belegen, und legt diese über den zugehörigen Blogeintrag in die Portfolioansicht. So werden verschiedene Kompetenznachweise («Pieces of Evidence») für das Curriculumsportfolio ausgewählt, wobei über das Reflexionsstatement jeweils die Verbindung zwischen Kompetenzbeschreibung, Lernziel, Lernergebnis, Lernprodukt und erhaltenes Feedback hergestellt wird.

5. «Presentation»: Die ausgewählten Kompetenznachweise werden übersichtlich zusammengestellt in einer Ansicht («View») präsentiert, wobei das Portfolio zusätzlich Informationen über den Eigentümer/die Eigentümerin enthält sowie einen Cover Letter, in dem das Zielpublikum über den Zweck und den Kontext der Portfolioerstellung informiert wird. Ein Statement zur Selbstbewertung der 
eigenen Leistung in diesem Modul schliesst das Curriculumsportfolio ab. Die Lerngruppe sowie die Vortragenden erhalten Zugriff auf die Portfolioansicht und können Kommentare abgeben.

Die Reflexion als zentrales Element erfolgt dabei über den gesamten Portfolioprozess hinweg: einerseits zu einzelnen Lernprodukten vor, während und nach deren Erstellung, andererseits in einem bewertenden Abschlussstatement (Barrett \& Carney 2005, S. 1). Wichtiges Ziel ist dabei, dass die Selbstbewertung der Lernergebnisse mit der Fremdbewertung durch Peers und Lehrende quasi «in einen Dialog» tritt, aus dem sich wertvolle neue Erkenntnisse ergeben.

\section{Assessment und Peer-Review}

Portfolios können grundsätzlich auf zwei verschiedene Arten bewertet werden (Biggs 2003, S. 19):

1. Bewertung der einzelnen Artefakte und anschliessende Kombination der Einzelwertungen

2. Bewertung des Portfolios in seiner Gesamtheit

Im Lehrgang «MA eEducation» kommen beide Varianten zum Einsatz. Die Wahl hängt von der didaktischen Konzeption und den Aufgabenstellungen ab und vari-

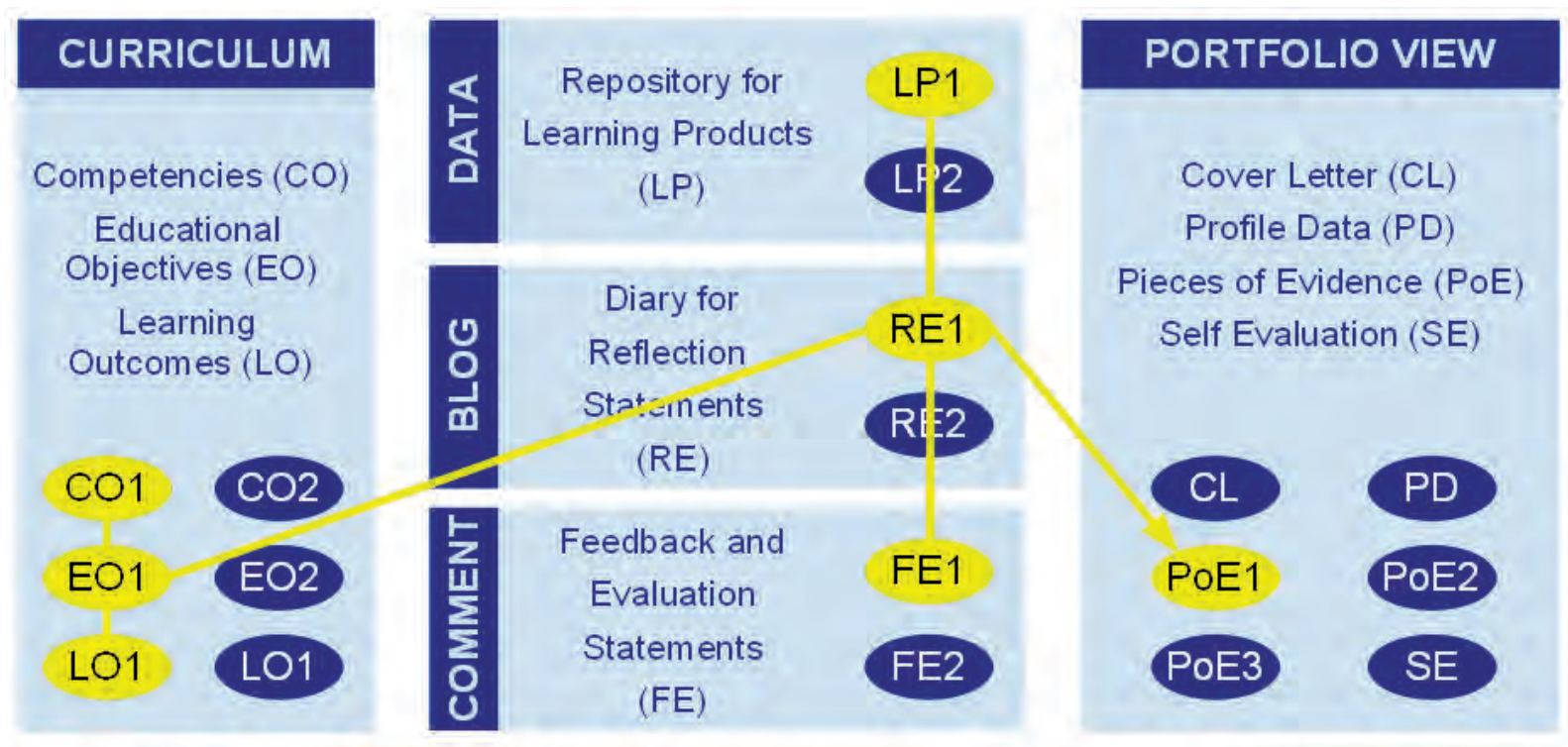

Context Definition

\section{Collection}

Reflection

Selection

Presentation

Abbildung 9: Darstellung des Curriculumsportfolios im Lehrgang «MA eEducation» 
iert von Modul zu Modul, wobei in jedem Fall das summative Assessment am Ende des Moduls anhand der erstellten Portfolioansicht vorgenommen wird - unabhängig davon, auf welche Art die Gesamtnote für das Modul gebildet wird.

Portfolios können sowohl für summative als auch für formative Assessmentzwecke verwendet werden (Cooper \& Love 2001, S. 1). Obwohl das Curriculumsportfolio im Lehrgang "MA eEducation» in erster Linie an den Lernergebnissen und den Kompetenznachweisen orientiert und somit eher dem summativen, produktorientierten Typ zuzuordnen ist, erfolgt auch ein formatives "Assessment for Learning" als begleitendes Feedback zum Reflexionsportfolio. Abbildung 10 stellt summative und formative Aspekte bei der Portfolioarbeit gegenüber:

Zwischenfeedback erfolgt im Laufe des Moduls auf drei Arten:

1. Lehrende geben zu bestimmten Abgabeterminen ein wertendes Feedback auf einzelne Lernprodukte ab und ermöglichen eine Überarbeitung für die endgültige Portfolioansicht zum Modulende.

2. Peers begleiten sich gegenseitig über das Reflexionsportfolio in einem «Einszu-eins-Coaching» bis zum fertigen Curriculumsportfolio. Die gebildeten Paare für das Coaching werden von der Lehrgangsleitung eingeteilt und wechseln von Modul zu Modul, so dass alle Studierenden mindestens einmal in Kontakt kommen.

3. Die Peers bewerten die erstellten Curriculumsportfolioansichten vor der endgültigen Abgabe bei den Lehrenden.

Die unter 3. genannte Bewertung der gesamten Portfolioansicht ist unabhängig von einer Bewertung nach fachlich-inhaltlichen Kriterien durch die Lehrenden und erfolgt in drei Kriterienbereichen, die zusammen mit dem Studierenden entwickelt wurden:

1. Intensität - Authentizität - Reflexivität:

Diese Kategorie soll sicherstellen, dass das Portfolio keine oberflächliche Sammlung von «Produkten» wird, die schlimmstenfalls nicht von den Lernenden selbst stammen könnten (vgl. hierzu Bräuer 2006; Häcker 2005). In den Reflexionen soll eine vertiefte, durchaus kritische Auseinandersetzung mit den dargebotenen Modulthemen und -inhalten deutlich sowie Probleme, Lösungsansätze und Erfolge beim individuellen Lernprozess sichtbar werden. Diese Kategorie betont den Prozesscharakter des Portfolios.

2. Dokumentation der Kompetenznachweise:

Im Gegensatz zu 1. steht hier das Produktportfolio im Vordergrund. Die abgelegten Kompetenznachweise sollen in kompakter, übersichtlicher Form die wesentlichen Lernerfolge des Moduls belegen und die erworbenen Kompetenzen veranschaulichen (Cooper \& Love 2001, S. 3). 
3. Ästhetik - Multimediale Aufbereitung - Kreativität:

Ein Portfolio zeigt eine Auswahl der besten Arbeiten, die im Zuge des Lernprozesses mit besonderer Hingabe erstellt und gestaltet wurden und deshalb einen hohen Wert für die Lernenden haben. Gemäss dem Ursprung des Begriffes «Portfolio» sollen die Artefakte und deren Einbettung in das Portfolio die Betrachter/innen erfreuen und das kreative, gestalterische und künstlerische Potential des Erstellers/der Erstellerin spürbar werden lassen. In einer elektronischen Form gehört zu dieser Kategorie insbesondere die multimediale Aufbereitung und Gestaltung und das Ansprechen verschiedener Sinne beim Betrachter/bei der Betrachterin.

Nach dem Peer-Feedback haben die Studierenden die Möglichkeit, ihr Portfolio vor der Endabgabe und der Abschlussbeurteilung noch einmal zu überarbeiten. Die Bewertung in den drei Kategorien ist insbesondere zu Beginn des Studiums eine wertvolle Stütze, wenn die Portfolioprozesse allen Lernenden noch nicht vertraut sind - mit zunehmender Dauer nimmt deren Bedeutung ab. Die Peer-Review im «Eins-zu-eins-Coaching» ist verpflichtend und wird durch die Einrechnung in die Workload des Moduls über ECTS anerkannt, fliesst aber nicht in die Modulnoten ein. In der Lerngruppe ist vereinbart, dass das Feedback für alle sichtbar abgegeben wird - nach Abschluss des Moduls können die Portfolioeigentümer/ innen selbst entscheiden, ob das Feedback weiterhin sichtbar bleiben soll.

Die Beurteilungskriterien für die fachlich-inhaltliche Bewertung der Portfolios durch die Vortragenden werden für jedes Modul - angepasst an dessen Lernziele und Lernergebnisse - individuell gebildet. Sie beziehen sich zumeist auf einzelne Artefakte und variieren sehr stark von Modul zu Modul, je nach Lernaktivität und gewünschtem Lernergebnis, so dass deren Beschreibung den Rahmen dieses Artikels sprengen würde. Die Kriterien sind jedenfalls zu Beginn der Portfolioarbeit den Lernenden bekannt, werden in der Lerngruppe diskutiert und teilweise auch weiterentwickelt.

\section{Das Präsentationsportfolio}

Zu Beginn des Studiums erstellen die Studierenden eine erste Portfolioansicht, die «Steckbrief» genannt wird, und präsentieren sich darin den Peers und den Lehrenden. Die Steckbriefe enthalten persönliche Daten, ein Bild, einen Cover Letter sowie einen tabellarischen Lebenslauf vergleichbar dem Europass-Lebenslauf, wobei die Studierenden selbst entscheiden, wie viel sie von sich in der Gruppe preisgeben. Mit dem Absolvieren der einzelnen Module des Curriculums entsteht im Verlauf des Studiums eine Sammlung von Portfolioansichten, die zusammen mit 


\section{Portfolio für summative Bewertungen} des Lernens

\section{Portfolio zur formativen Bewertung zur Unterstützung des Lernens}

Der Zweck des Portfolios wurde von einer Institution vorgeschrieben.

Artefakte werden von Institutionen vorgeschrieben um die Ergebnisse einer Instruktion festzulegen.

Portfolios werden üblicherweise am Ende eines Schuljahres, Semesters oder Programms angefertigt, mit Zeitbeschränkung-

Die Portfolios und/oder Artefakte werden üblicherweise benotet, basierend auf einer Matrix und quantitativen Daten für ein externes Publikum-

Das Portfolio ist üblicherweise durch die vorgegebenen Ergebnisse, Ziele oder Standards strukturiert.

Manchmal werden sie benutzt, um wichtige Entscheidungen zu treffen-

Summativ - was wurde bis heute gelernt? (Vergangenheit - Gegenwart)

extrinsische Motivation ist notwendig

Publikum: extern, geringe Auswahlmöglichkeiten
Der Zweck des Portfolios wurde mit dem/der Lernenden abgestimmt.

Artefakte wurden vom Lerner/von der Lernerin ausgewählt ,um damit die Geschichte ihres Lernens zu erzählen

Portfolios werden laufend gepflegt, über ein Schuljahr, Semester oder Programm hinweg, mit flexibler Zeiteinteilung-

Die Portfolios und Artifakte werden mit den Lerneden begutachtet und benutzt, um Rückmeldung zur Verbesserung des Lernens zu geben.

Die Organisation des Portfolios ist durch den Lernenden bestimmt oder mit dem Mentor/Berater/Lehrer ausgehandelt

Sie werden kaum genutzt, um wichtige Entscheidungen zu treffen.

Formativ - welche Lernbedürfnisse gibt es in der

Zukunft? (Gegenwart - Zukunft)

hegt intrinsische Motiation, mobilisiert den/die Lernenden

Publikum: Lernende, Familie, Freunde - Lernende können sie auswählen

Abbildung 10: Gegenüberstellung von summativem und formativem Assessment mit Hilfe von E-Portfolios nach Barrett und Carney (2005, S. 6), übertragen ins Deutsche von Hornung-Prähauser et al. (2007, S. 19)

dem Steckbrief als «Portfoliostartseite» als umfangreiches Präsentationsportfolio auch ausserhalb des Studiums verwendet werden kann ${ }^{6}$.

\section{Evaluation und Weiterentwicklung}

Die bisherigen sehr positiven Erfahrungen nach dem Pilotversuch und etwa einem Jahr Vollimplementierung werden in diesem Studienjahr in einem begleitenden Forschungsprojekt einer umfangreichen Evaluation unterzogen. Neben der Verbesserung des E-Portfoliokonzepts ist auch ein Re-Design der kompletten Lernumgebung durch die Implementierung eines Studierendenportals geplant, das

\footnotetext{
6 Die Software ermöglicht hierfür eine differenzierte Zugriffsregelung innerhalb der Plattform, aber auch ganz öffentlich im Sinne einer Website. Darüber hinaus ist es möglich, nicht-registrierten Mahara-Usern und -Userinnen einen Einblick in das Portfolio über einen individuellem Zugangsschlüssel zu erlauben, so dass das Portfolio vor Suchmaschinen verborgen bleibt (vgl. hierzu die Roadmap auf http://www.mahara.org.)
} 
die diversen verwendeten Dienste und Plattformen (Moodle, Mahara, Wiki etc.) unter einem Dach vereint.

\section{Literatur}

Anderson, Lorin W.; Krathwohl, David R. (2001). A taxonomy for learning, teaching, and assessing: a revision of Bloom's taxonomy of educational objectives. New York: Addison Wesley Longman.

Arbeitsstelle für Hochschuldidaktik der Universität Zürich (2007). Leistungsnachweise in modularisierten Studiengängen. http://www.afh.uzh.ch/instrumente/dossiers/Leistungsnachweise_Juli_07.pdf (16.04.2010).

Attwell, Graham (2007). «Personal Learning Environments - the future of eLearning?» eLearning Papers, Vol. 2 No. 1: 1-8. http://www.elearningeuropa.info/files/media/media11561. pdf (16.04.2010).

Ayala, Javier I. (2006). «Electronic Portfolios for Whom?» Educause Quarterly, Number 1 2006: 12-13. http://net.educause.edu/ir/library/pdf/egm0613.pdf (16.4.2010).

Bacher, Gottfried (2006). «Empfehlung der nationalen Bologna Follow-up Gruppe für die modulare Gestaltung der Lehrpläne sowie für die Aussetzung der ECTS-grading scale.» http://www.bmwf.gv.at/fileadmin/user_upload/europa/bologna/empfehlung_lehrpl.pdf (16.04.2010).

Bakic, Josef (2006). «ECTS.»Pädagogisches Glossar der Gegenwart - Von Autonomie bis Zertifizierung. Hrsg. v. Agnieszka Dzierzbicka u. Alfred Schirlbauer, 2. Aufl. Wien: Löcker. 105-113.

Barrett, Helen C.; Carney, Joanne (2005). Conflicting Paradigms and Competing Purposes in Electronic Portfolio Development. http://electronicportfolios.org/portfolios/LEAJournal-BarrettCarney.pdf (16.4.2010).

Baumgarnter, Peter; Himpsl, Klaus; Zauchner, Sabine (2009). Einsatz von E-Portfolios an (österreichischen) Hochschulen: Zusammenfassung - Teil I des BMWF-Abschlussberichts «E-Portfolio an Hochschulen»: GZ 51.700/0064-VII/10/2006. Forschungsbericht. Krems: Department für Interaktive Medien und Bildungstechnologien, Donau Universität Krems. http://www.peter.baumgartner.name/Members/baumgartner/news/e-portfolioforschungsbericht-und-publikationsstrategie/ (16.04.2010).

Baumgartner, Peter (2005). «Eine neue Lernkultur entwickeln: Kompetenzbasierte Ausbildung mit Blogs und E-Portfolios.» ePortfolio Forum Austria 2005. Hrsg. v. Veronika Hornung-Prähauser. Salzburg, Österreich. 33-38. http://edumedia.salzburgresearch. at/images/stories/EduMedia/1_EduMedia_2005/eportfolio_tagungsband_deutsch_final_25042005.pdf (01.12.2009).

Baumgartner, Peter (2008). "Blended Learning Arrangements. » ; ,E-Learning \& Wissensmanagement Jahrbuch 2008. Hrsg. v. Uwe Beck, Winfried Sommer, Frank Siepmann. Karlsruhe: KKA. 10-17.

Baumgartner, Peter (2008). Eine Taxonomie für E-Portfolios - Teil II des BMWF-Abschlussberichts «E-Portfolio an Hochschulen»: GZ 51.700/0064-VII/10/2006. Forschungsbericht. Krems: Department für Interaktive Medien und Bildungstechnologien, Donau Universität Krems.

Biggs, John (2003). Teaching For Quality Learning at University. 2. Aufl. Buckingham: Open University Press. 
Binder, Ulrich (2006). «Modularisierung. »Pädagogisches Glossar der Gegenwart - Von Autonomie bis Zertifizierung. Hrsg. v. Agnieszka Dzierzbicka u. Alfred Schirlbauer. 2. Aufl. Wien: Löcker. 183-190.

Bräuer, Gerd (2006). «Keine verordneten Hochglanz-Portfolios, bitte! Die Korruption einer schönen Idee? » Das Handbuch Portfolioarbeit. Hrsg. v. Ilse Brunner, Thomas Häcker, Felix Winter. Hannover: Friedrich. 257--261.

Brunner, Ilse; Häcker, Thomas; Winter, Felix (2006). Das Handbuch Portfolioarbeit. Hannover: Friedrich.

Buzzetto-More, Nicole A.; Alade, Ayodele (2008). «The Pentagonal E-Portfolio Model for Selecting, Adopting, Building, and Implementing an E-Portfolio.» Journal of Information Technology Education. 7. 184-208. http://jite.org/documents/Vol7/JITEv7IIP045-070More383.pdf (01.12.2009).

Cooper, Trudi; Love, Terence (2001). «Online portfolios: issues of assessment and pedagogy.» AARE 2001: Crossing borders: New frontiers of educational research. Hrsg. v. Peter Jeffrey. Coldstream, Victoria: AARE Inc. 417--426. http://www.aare.edu.au/01pap/ coo01346.htm (1.12.2009).

Erpenbeck, John; Sauter, Werner (2007). Kompetenzentwicklung im Netz: New Blended Learning mit Web 2.0. Köln: Luchterhand (Hermann).

Forehand, Mary (2005). «Bloom's Taxonomy - Original and revised.» Emerging perspectives on learning, teaching, and technology. Hrsg. v. Michael Orey. http://projects.coe.uga. edu/epltt/index.php?title=Bloom\%27s_Taxonomy (1.12.2009).

Häcker, Thomas (2005). Portfolio als Instrument der Kompetenzdarstellung und reflexiven Lernprozesssteuerung. http://www.bwpat.de/ausgabe8/haecker_bwpat8.pdf (1.12.2009).

Häcker, Thomas (2007). Portfolio: ein Entwicklungsinstrument für selbstbestimmtes Lernen: Eine explorative Studie zur Arbeit mit Portfolios in der Sekundarstufe I. Hohengehren: Schneider Verlag.

Himpsl, Klaus; Baumgartner, Peter (2009). Evaluation von E-Portfolio-Software - Teil III des BMWF-Abschlussberichts «E-Portfolio an Hochschulen»: GZ 51.700/0064-VII/10/2006. Forschungsbericht. Krems: Department für Interaktive Medien und Bildungstechnologien, Donau Universität Krems. http://www.bildungstechnologie.net/blog/evaluationvon-e-portfolio-software-abschlussbericht (1.12.2009).

Hornung-Prähauser, Veronika; Geser, Guntram; Hilzensauer, Wolf; Schaffert, Sandra (2007). Didaktische, organisatorische und technologische Grundlagen von E-Portfolios und Analyse internationaler Beispiele und Erfahrungen mit E-Portfolio-Implementierungen an Hochschulen. Salzburg: Salzburg Research Forschungsgesellschaft. http://edumedia. salzburgresearch.at/images/stories/e-portfolio_studie_srfg_fnma.pdf (1.12.2009).

Ravet, Serge (2007). E-Portfolio Position Paper. http://www.eife-l.org/publications/eportfolio/documentation/positionpaper (1.12.2009).

Reinmann-Rothmeier, Gabi (2003). Didaktische Innovation durch Blended Learning: Leitlinien anhand eines Beispiels aus der Hochschule. Bern: Huber.

Sankofi, Martin; Szucsich, Petra (2007). Blended Learning Modelle in der universitären Ausbildung am Fallbeispiel einer zertifizierbaren Fortbildungsmaßnahme für FremdsprachenlehrerInnen an der Donau-Universität Krems. Master Thesis, Donau-Universität Krems. http://szucsich.sankofi.com/Masterthese_Sankofi_Szucsich_02_10_07.pdf (1.12.2009).

Sauter, Werner; Sauter, Annette; Bender, Harald (2004). Blended Learning. Effiziente Integration von E-Learning und Präsenztraining. 2. Aufl. Köln: Luchterhand (Hermann). 
Veugelers, Marij; Aalderink, Wijnand (2006). Portfolio models and community building in dutch higher education, lessons learned of $\mathrm{nl}$ portfolio. http://www.eife-l.org/publications/eportfolio/proceedings2/ep06/ep2006_papers/veuglers/ (1.12.2009). 